\title{
Evaluation of the anti-allergic activity of Citrus unshiu using rat basophilic leukemia RBL-2H3 cells as well as basophils of patients with seasonal allergic rhinitis to pollen
}

\author{
SHOKO KOBAYASHI ${ }^{1}$ and SOICHI TANABE ${ }^{2}$ \\ ${ }^{1}$ Department of Food and Life-Science, Takasaki University of Health and Welfare, Takasaki 370-0033; ${ }^{2}$ Graduate School of \\ Biosphere Science, Hiroshima University, 1-4-4 Kagamiyama, Higashi-Hiroshima, Hiroshima 739-8528, Japan
}

Received October 10, 2005; Accepted November 18, 2005

\begin{abstract}
The anti-allergic activity of the 50\% methanol extract of Citrus unshiu powder (MEC) was examined. Fifty percent methanol extract of MEC powder showed potent inhibitory activity against histamine release from basophils of patients suffering from seasonal allergic rhinitis to ceder pollen. To examine this anti-allergic mechanism in detail, we next used rat basophlilic leukemia RBL-2H3 cells. MEC significantly inhibited IgE-induced histamine and Bhexosaminidase release from RBL-2H3 cells. Since MEC contains a variety of flavonoids, we selected nobiletin, hesperetin, and hesperidin (hesperetin glycoside) as representative compounds, and further evaluated these inhibitory activities. Among the flavonoids tested, hesperetin was the most potent, while hesperidin had far less, if any, inhibitory activity. The mechanism by which flavonoids inhibited the degranulation process was then examined. As a result, hesperetin and nobiletin suppressed the phosphorylation of Akt-1, direct downstream effector of phosphatidylinositol 3-kinase (PI3-K). Thus, it was assumed that these flavonoids suppressed IgEmediated stimulation of basophils through PI3-K pathway and that proper intake of Citrus unshiu would be favorable for managing seasonal allergic rhinitis to ceder pollen.
\end{abstract}

Correspondence to: Dr Soichi Tanabe, Graduate School of Biosphere Science, Hiroshima University, 1-4-4 Kagamiyama, Higashi-Hiroshima, Hiroshima 739-8528, Japan

E-mail: stanabe@hiroshima-u.ac.jp

Abbreviations: DMSO, dimethyl sulfoxide; DNP, dinitrophenyl; ELISA, enzyme-linked immunosorbent assay; HSA, human serum albumin; MEC, 50\% methanol extract of Citrus unshiu powder; MT, modified Tyrode buffer; PBMC, peripheral blood mononuclear cell; PCA, passive cutaneous anaphylaxis; PI3-K, phosphatidylinositol 3-kinase; RBL-2H3, rat basophil leukemia; SAR, seasonal allergic rhinitis

Key words: anti-allergic activity, rhinitis to pollen, Citrus unshiu, basophils, hesperetin, nobiletin, Akt

\section{Introduction}

Allergic rhinitis is a common allergic disease in industrialized nations, characterized by sneezing, water rhinorrhea, itching in the nose, eyes, and palate, and nasal congestion (1). Recently, the incidence of both perennial (2-5) and seasonal allergic rhinitis (6-10) has been increasing worldwide. Seasonal allergic rhinitis (SAR) may occur at any age and affects between 10 and $30 \%$ of children (6). It causes major discomfort in affected patients, such as fatigue, absenteeism, and irritability, adversely affecting the child's adjustment and achievement at school. Therefore, effective management should be directed not only towards rapid and adequate relief of the clinical symptoms but also towards a sustained improvement in the patient's daily life. While conventional therapies for allergic symptoms are advancing steadily, natural products such as phytochemicals and herbal extracts have been widely used by consumers as alternatives to prescription drugs without definitive clinical evidence.

Recently, citrus flavonoids have been reported to possess various pharmacological effects, such as anti-inflammation and even anti-cancer activity (11-13). For these activities, nobiletin, a polymethoxy (5, 6, 7, 8, 3', 4'-hexamethoxy) flavone, is thought to be a responsible compound and has been paid much attention. Concerning the anti-allergic effect of citrus flavonoids, hesperidin is the most famous one. Park et al reported that orally administrated hesperidin, flavanone glycoside, inhibited the passive cutaneous anaphylaxis (PCA) reaction of mice, but exhibited no PCA-inhibitory activity when administered intraperitoneally (14). Another group also demonstrated that hesperidin did not inhibit histamine release in vitro (15). However, hesperetin, the flavanone aglycone, inhibited $\beta$-hexosaminidase release in vitro (14). The antiallergic activities of hesperidin and hesperetin are controversial since the mechanisms by which these citrus flavonoids exert their activities have not been fully clarified yet. In addition, to our knowledge, the anti-allergic activity of nobiletin has not been examined in detail.

For this study, we chose Citrus unshiu, belonging to the family, Rutaceae. It is one of the most popular citrus fruits and its well-known name is Satsuma Mandarin. The ripe fruits are a common food and the dried peels have been used not only in traditional Chinese medicine but also as a skinmoisturizing agent in Japanese folk medicine (16). Citrus 
unshiu has been reported to contain several biologically active components, such as auraptene, ß-cryptoxanthin, limonine, hesperidin, nobiletin and tangeretin $(11,17)$.

To evaluate the anti-allergic activity of Citrus unshiu, basophils of patients with SAR to Japanese cedar pollen were kindly donated and used in the first part of the present study. We also used the rat basophilic leukemia cell line, RBL-2H3, which has been widely used to examine signal transduction (18). We show that Citrus unshiu inhibited the chemical mediator release from patients' basophils and from the rat basophilic cell line. We also described the anti-allergic activity of nobiletin and hesperetin and demonstrated that the inhibitory effect was caused, at least in part, by the inhibition of phosphatidylinositol 3-kinase.

\section{Materials and methods}

Materials. Citrus unshiu powder and Japanese ceder (Cryptomeria japonica) pollen extract were obtained from Fuji Flour Milling (Shizuoka, Japan) and Hayashibara Biochemical Laboratories (Okayama, Japan), respectively. For assays (Figs. 1 and 2), 50\% methanol extract of Citrus unshiu powder (MEC) was used. The concentrations in the Figs. are expressed as the MEC equivalent. Wortmannnin was purchased from Sigma-Aldrich (St. Louis, MO). All other chemicals were of reagent grade.

Patients. Whole blood samples (approximately $5 \mathrm{ml}$ ) were donated from 4 patients with SAR to Japanese cedar pollen. The definition of pollen allergy was made on the basis of an episode of allergic reaction combined with positive supportive laboratory data such as IgE-ELISA. Informed consent was obtained from all donors.

Histamine release in human basophils. For histamine release assay, leukocyte fractions were prepared by dextran sedimentation (19) using the individual whole blood samples. Histamine release was assayed as described previously $(19,20)$. Briefly, basophils from the above mentioned leukocytes were suspended in modified Tyrode buffer (MT, $100 \mu \mathrm{l}$ ) and incubated at $37^{\circ} \mathrm{C}$ for a few minutes. Japanese ceder pollen extract $(0.1 \mu \mathrm{g} / \mathrm{ml})$ was added to the leukocytes, and the effects of the addition of MEC $(1.6 \mathrm{mg} / \mathrm{ml})$ or wortmannin (positive control, $25 \mu \mathrm{M}$ ) were examined. Wortmannin was dissolved in dimethyl sulfoxide (DMSO) and diluted with MT; the final DMSO concentration was $0.1 \%$. The mixtures were incubated at $37^{\circ} \mathrm{C}$ for $40 \mathrm{~min}$ and then centrifuged at $400 \mathrm{x} \mathrm{g}$ for $5 \mathrm{~min}$. Histamine release in each supernatant was quantified by HPLC according to the on-column conversion method by using $o$-phthalaldehyde (21). The results are expressed as the percentage release of histamine amount by pollen extract alone.

Inhibitory activity measurement of MEC on RBL-2H3 cells. The inhibitory activity of MEC and wortmannin against the release of histamine and $B$-hexosaminidase from RBL-2H3 cells was evaluated according to the method reported by Watanabe et al (22). RBL-2H3 cells were purchased from Health Science Research Resource Bank (Japan). Dulbecco's modified Eagle's medium (DMEM, Invitrogen, Carlsbad, CA) containing $10 \%$ heat-inactivated fetal calf serum (Invitrogen)

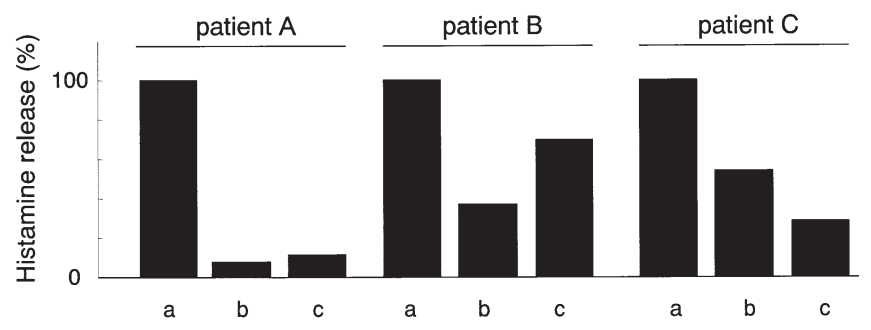

Figure 1. Effects of MEC on the IgE-induced histamine release from the patients' basophils. Japanese ceder pollen extract $(0.1 \mu \mathrm{g} / \mathrm{ml})$ was added to the basophils, and the resulting histamine release in the supernatant was quantified by HPLC. The results are expressed as the percentage release of histamine amount by pollen extract alone (control). a, control; b, MEO $(1.6 \mathrm{mg} / \mathrm{ml})$; c, wortmannin $(25 \mu \mathrm{M})$.

was used as a growth medium. The cells were cultured in a 24-well cell culture plate $\left(2.5 \times 10^{5} /\right.$ well, Becton-Dickinson Co., Franklin Lakes, NJ) overnight at $37^{\circ} \mathrm{C}$ under a humidified $5 \% \mathrm{CO}_{2}$ atmosphere. The cells were incubated in growth medium containing $50 \mathrm{ng} / \mathrm{ml}$ of mouse monoclonal antidinitrophenyl (DNP) IgE for $2 \mathrm{~h}$. After washing with MT, MEC $(1.6,8$, or $16 \mathrm{mg} / \mathrm{ml})$ or wortmannin $(25 \mu \mathrm{M})$ was added. Wortmannin was dissolved in DMSO and diluted with MT; the final DMSO concentration was $0.1 \%$. After $10 \mathrm{~min}$ of incubation, DNP-labeled human serum albumin (DNP-labeled HSA, $50 \mathrm{ng} / \mathrm{ml}$ final concentration) was added and the culture was incubated for $30 \mathrm{~min}$. The histamine concentration of the resulting supernatant of the cell culture was measured by HPLC using the method described above. The $\beta$-hexosaminidase activity of the supernatant was measured by using the method of Demo et al (23). Briefly, $50 \mu 1$ of the supernatant was mixed with $100 \mu 1$ of a $0.1 \mathrm{M}$ citrate buffer ( $\mathrm{pH} 4.5$ ) containing $3.3 \mathrm{mM} p$-nitrophenyl-2-acetoamide- $\beta$-D-glucopyranoside and the mixture was incubated in a $96-$ well plate at $37^{\circ} \mathrm{C}$ for $25 \mathrm{~min}$. The reaction was terminated by adding a $2 \mathrm{M}$ glycine buffer ( $\mathrm{pH} 10.4,100 \mu \mathrm{l})$, and absorbance at $405 \mathrm{~nm}$ was measured using a microplate reader (model 550, Bio-Rad, CA). The results are expressed as percentage release of histamine or ß-hexosaminidase activity amount by antigen (DNP-labeled HSA) alone.

Inhibitory activity measurement of flavonoids on RBL-2H3 cells and Akt phosphorylation. RBL-2H3 cells were plated at $2.5 \times 10^{6}$ cells per plastic dish $(100 \times 20 \mathrm{~mm}$, Corning, NY) in DMEM growth media (10\% heat-inactivated FCS) overnight. After washing with MT, flavonoids (100 or $500 \mu \mathrm{M}$ ) or wortmannin $(25 \mu \mathrm{M})$ were added. Each compound was dissolved in DMSO and diluted with MT. The final DMSO concentration was $0.1 \%$. After $10 \mathrm{~min}$ of incubation, DNPlabeled HSA (50 $\mathrm{ng} / \mathrm{ml}$ final concentration) was added and the culture was incubated for $30 \mathrm{~min}$. The resulting supernatant was collected, and the $\beta$-hexosaminidase activity and histamine concentration were measured using the abovementioned method. Cells were then washed twice with icecold PBS and lysed in $100 \mu 1$ of NP40 cell lysis buffer (BioSource International, Inc., Camarillo, CA) containing $1 \mathrm{mM}$ phenylmethylsulfonyl fluoride (Sigma-Aldrich) and $1 \mathrm{X}$ protease inhibitor cocktail (Sigma-Aldrich). The reaction was quenched by cooling in an ice bath for $30 \mathrm{~min}$. Lysates were clarified by centrifugation and the total protein 
A)

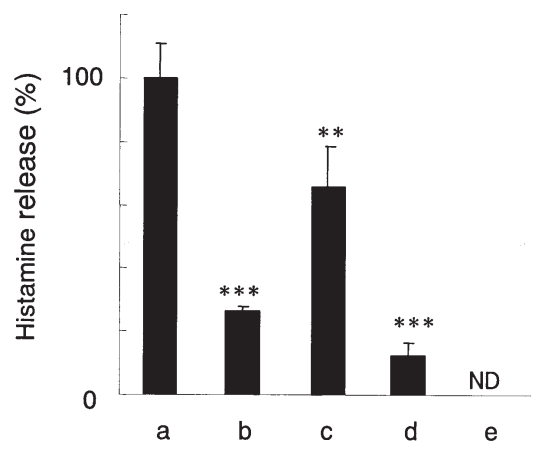

B)

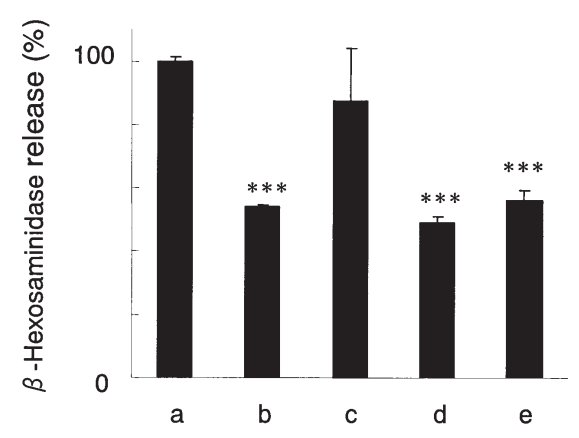

Figure 2. Effects of MEC on the IgE-induced histamine (A) and B-hexosaminidase (B) release from RBL-2H3 cells. Sensitized RBL-2H3 cells were challenged with DNP-labeled HSA, and the resulting histamine and B-hexosaminidase release in the supernatant was quantified. The results are expressed as percentage release of histamine or B-hexosaminidase activity amount by DNP-labeled HSA alone (control). a, control; b, wortmannin ( $25 \mu \mathrm{M})$; $\mathrm{c}$, MEO $(1.6 \mathrm{mg} / \mathrm{ml})$; d, MEO $(8 \mathrm{mg} / \mathrm{ml})$; e, MEO $(16 \mathrm{mg} / \mathrm{ml}) .{ }^{* *} \mathrm{p}<0.01 ;{ }^{* * *} \mathrm{p}<0.001$ in comparison with controls

concentrations were measured using Protein assay dye reagent (Bio-Rad). Each resulting supernatant was diluted to a final concentration of 200-400 $\mu \mathrm{g} / \mathrm{ml}$. Akt phosphorylation level was investigated by the micro-beads method using Luminex (Hitachi Software, Yokohama, Japan). Briefly, diluted lysates were incubated with Luminex beads conjugated with anti-Akt (total) and anti-phospho-Akt (Ser473) antibody (BioSource International, Inc.) followed by sandwich immunoassay using biotinylated secondary antibodies. Beads were washed three times during each incubation. Phycoerythrin-streptavidin was used as a developing reagent. The relative fluorescence units of Akt (total) and phospho-Akt were detected by counting 100 beads with Luminex apparatus. The data in Fig. 5 are expressed as percentage Akt phosphorylation by antigen (DNP-labeled HSA) alone, based on the calculation of phospho-Akt/Akt (total).

\section{Results}

MEC potently inhibited degranulation of basophils. Firstly, $50 \%$ methanol-soluble fraction from the Citrus unshiu powder (MEC) was prepared, and examined for inhibitory effects on histamine release by ceder pollen from four individual basophils of patients with SAR (Fig. 1). Unfortunately, PBMC from one patient was not reactive to ceder pollen exposure in this assay. For comparison, wortmannin was used as a positive control. MEC as well as wortmannin inhibited the histamine release from basophils of all three patients. The degree of inhibition was patient-dependent; MEC was more potent than wortmannin in patients $\mathrm{A}$ and $\mathrm{B}$ while wortmannin was more potent in patient $\mathrm{C}$ (Fig. 1).

Practically, the precise examination of degranulation inhibitory activity using basophils of patients is limited. Therefore, the inhibitory activity of MEC on histamine release from sensitized RBL-2H3 cells induced by antigen was examined (Fig. 2). A test sample is normally added to releasing medium containing cells challenged with the antigen (DNP-labeled HSA). In this case, we assume that the test sample has an effect downstream from the antigen-stimulated stage in the cascade of an immediate type of allergic reaction.

The inhibitory effect of MEC was concentration-dependent. The histamine release at $1.6 \mathrm{mg} / \mathrm{ml}$ of MEC was significantly<smiles>COc1ccc([C@@H]2CC(=O)c3c(OC)c(OC)c(OC)c(OC)c3O2)cc1OC</smiles><smiles>COc1ccc([C@@H]2CC(=O)c3c(O)cc(O)cc3O2)cc1O</smiles>

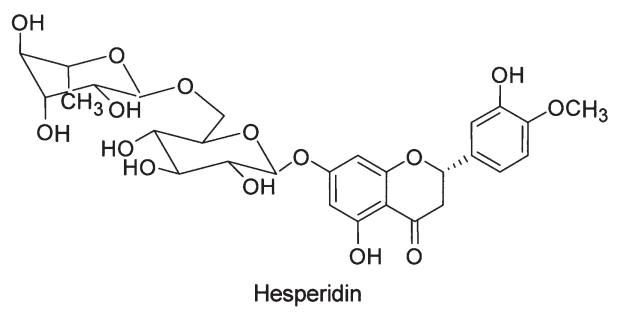

Figure 3. Structures of the flavonoids tested.

decreased compared to the control $(\mathrm{p}<0.01)$, and $16 \mathrm{mg} / \mathrm{ml}$ of MEC inhibited histamine release almost perfectly (Fig. 2A). Since $\beta$-hexosaminidase was frequently employed as a marker molecule for examining the degranulation process of mast cells (24), we also measured $\beta$-hexosaminidase release from RBL$2 \mathrm{H} 3$ to confirm the degranulation inhibitory effect of MEC (Fig. 2B). The inhibitory effect of MEC was concentrationdependent; the $\beta$-hexosaminidase release at $8 \mathrm{mg} / \mathrm{ml}$ of MEC was decreased compared to the control $(\mathrm{p}<0.01)$ and reached a plateau at $16 \mathrm{mg} / \mathrm{ml}$ of MEC, but no significant inhibitory effect was observed at $1.6 \mathrm{mg}$ of MEC. Judging from both histamine and $\beta$-hexosaminidase release, the inhibitory activity of MEC at around $8 \mathrm{mg} / \mathrm{ml}$ seemed to be roughly equal to that of wortmannin at $25 \mu \mathrm{M}$.

Degranulation inhibitory activity of flavonoids (nobiletin, hesperetin, and hesperidin) (Fig. 3). MEC contains a variety of flavonoids, including hesperetin, hesperidin (hesperetin glycoside) and nobiletin, according to HPLC analyses (data not shown). We selected these three flavonoids as representative compounds, and further evaluated their degranulation inhibitory effect. In this study, $500 \mu \mathrm{M}$ of nobiletin significantly inhibited both histamine $(\mathrm{p}<0.01)$ and $\beta$-hexosaminidase 
A)

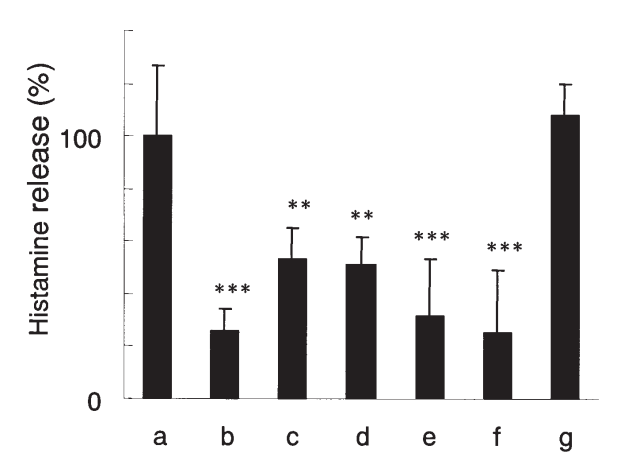

B)

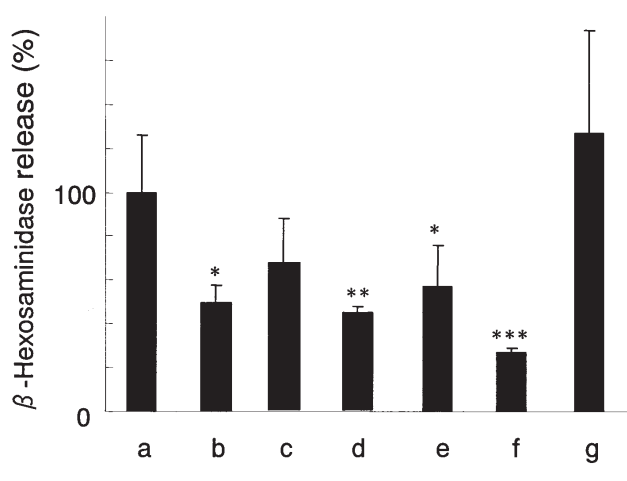

Figure 4. Effects of nobiletin, hesperetin, and hesperidin on the IgE-induced histamine (A) and B-hexosaminidase (B) release from RBL-2H3 cells. The experiment was performed as in Fig. 2. a, control; b, wortmannin $(25 \mu \mathrm{M})$; c, nobiletin $(100 \mu \mathrm{M})$; d, nobiletin $(500 \mu \mathrm{M})$; e, hesperetin (100 $\mu \mathrm{M})$; f, hesperetin $(500 \mu \mathrm{M}) ; \mathrm{g}$, hesperidin $(500 \mu \mathrm{M}) .{ }^{*} \mathrm{p}<0.05 ;{ }^{* *} \mathrm{p}<0.01 ;{ }^{* * *} \mathrm{p}<0.001$ in comparison with controls.

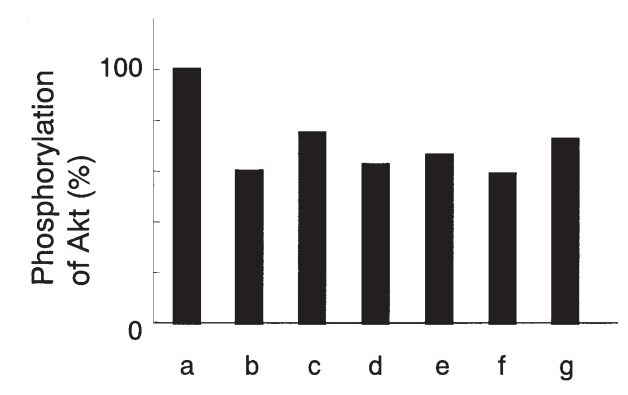

Figure 5. Effects of nobiletin, hesperetin, and hesperidin on the Akt phosphorylation of RBL-2H3 cells. Sensitized RBL-2H3 cells were challenged with DNP-labeled HSA, and the Akt phosphorylation level of the cell lysate was investigated by the micro-beads method using anti-Akt (total) and anti-phospho-Akt antibodies. The data are expressed as percentage Akt phosphorylation by DNP-labeled HSA alone (control), based on the calculation of phospho-Akt/Akt (total). a, control; b, wortmannin $(25 \mu \mathrm{M})$; $\mathrm{c}$, nobiletin $(100 \mu \mathrm{M}) ; \mathrm{d}$, nobiletin $(500 \mu \mathrm{M})$; e, hesperetin $(100 \mu \mathrm{M}) ; \mathrm{f}$, hesperetin $(500 \mu \mathrm{M}) ; \mathrm{g}$, hesperidin $(500 \mu \mathrm{M})$.

$(\mathrm{p}<0.01)$ release (Fig. 4). Hesperetin showed more potent activity; $100 \mu \mathrm{M}$ of hesperetin significantly inhibited both histamine $(\mathrm{p}<0.001)$ and $\beta$-hexosaminidase $(\mathrm{p}<0.05)$ release. However, hesperidin (hesperetin glycoside) had no effect on degranulation.

Suppression of PI3-K activity by nobiletin and hesperetin. The mechanism by which nobiletin and hesperetin inhibited the degranulation process of RBL-2H3 cells was then examined. Since it has been demonstrated that PI3-K is involved in the IgE-mediated stimulation of basophils (25), we examined the involvement of Akt, a serine/threonine kinase and a direct downstream effector of PI3-K (26). Akt phosphorylation was suppressed by wortmannin $(25 \mu \mathrm{M})$, nobiletin $(500 \mu \mathrm{M})$ and hesperetin $(500 \mu \mathrm{M})$ almost equally (Fig. 5). Unexpectedly, hesperidin $(500 \mu \mathrm{M})$ also suppressed (but weakly) the phosphorylation. Although phosphorylation of JNK (c-Jun N-terminal stress kinase) and p38 MAPK (mitogen-activated protein kinase) was measured using Luminex-micro-beads at the same time, the obtained data were not explicable (data not shown).

\section{Discussion}

Many studies on anti-allergic food compounds have been employed using animal models such as PCA reaction and/or animal cells such as RBL-2H3. The methods using PCA reaction and RBL-2H3 are well-established. In this study, however, basophils of patients with SAR to Japanese cedar pollen were used to demonstrate the anti-allergic activity of Citrus unshiu towards patients directly because it is well known that there are differences in sensitivity to anti-allergic compounds among patients. As shown in Fig. 1, there were differences in sensitivity to MEC and wortmannin between patients $\mathrm{C}$ and $\mathrm{A}$ or $\mathrm{B}$. Therefore, MEC might be more effective for patients A and B than C (Fig. 1). Patient basophil assay would be an excellent tool for predicting the effectiveness of an anti-allergic compound before its administration. On the other hand, since an animal cell line such as RBL-2H3 can be used without limit, it was favorable for the evaluation of the mechanisms by which MEC and flavonoids exert antiallergic effects.

We measured the histamine and $B$-hexosaminidase release from RBL-2H3 cells, since the enzyme is stored in the secretory granules of mast cells and is released together with such chemical mediators as histamine when mast cells are immunologically activated. As shown in Fig. 4, the release of B-hexosaminidase responded more drastically and dosedependently to flavonoid treatment than that of histamine.

According to the data of B-hexosaminidase (Fig. 4B), it was found that hesperetin $(100 \mu \mathrm{M})$ had a most potent degranulation inhibitory activity. Comparing hesperetin (aglycone, Fig. 3) and hesperidin (glycoside, Fig. 3), it was clearly found that only hesperetin had degranulation inhibitory activity in our assay (Fig. 4). This fact is well correlated with the data of Park et al (14). According to HPLC analyses, the major flavonoid occurring in Citrus unshiu was hesperidin, while the concentration of hesperetin was low (data not shown). However, hesperidin can be easily metabolized to hesperetin in the intestinal tract $(14,27)$. Thus, hesperetin and hesperidin in Citrus unshiu have anti-allergic activity when administered orally (28). Hesperetin is mainly absorbed as an intact form and is detected in both plasma and urine $(14,27)$, whereas 
most flavonoids and their glucosides are glucuronidated and metabolized during transfer across the intestine or the liver (29).

Another notable observation was that hesperidin weakly suppressed Akt phosphorylation (Fig. 5). Further analyses on other signal transduction pathways in which flavone aglycone and glycoside are involved could explain this discrepancy.

Although hesperidin and hesperetin are studied frequently as far as anti-allergic citrus flavonoids are concerned, we conclude that nobiletin should be included. Also, Park et al reported that naringenin exhibited more potent degranulation inhibitory activity than hesperetin (14). Therefore, comprehensive analyses are necessary to clarify whether other flavonoids (apart from the above-mentioned) in MEC also have this kind of activity.

In conclusion, we have provided direct evidence that Citrus unshiu powder inhibits histamine release from basophils of patients suffering from SAR to ceder pollen. Among the flavonoids in Citrus unshiu powder, hesperetin and nobiletin inhibited the degranulation of RBL-2H3 cells via, at least in part, suppression of PI-3 kinase activity. Therefore, citrus fruit (such as Citrus unshiu) powder would be a useful antiallergic therapy as part of a daily regime, and hesperetin and nobiletin might be therapeutic agents for treating IgE-mediated allergic disorders. A pilot clinical test to apply Citrus unshiu powder to the prevention of SAR is now being planned.

\section{References}

1. Thomet OAR, Schapowal A, Heinisch IVWM, Wiesmann UN and Simon H-U: Anti-inflammatory activity of an extract of Petasites hybridus in allergic rhinitis. Int Immunopharmacol 2: 997-1006, 2002.

2. Hu G, Walls RS, Bass D, Bullock R, Grayson D, Jones M and Gebski V: The Chinese herbal formulation Biminne in management of perennial allergic rhinitis: a randomized, double-blind, placebo-controlled, 12-week clinical trial. Ann Allergy Asthma Immunol 88: 478-487, 2002.

3. Chervinsky P, Casale T, Townley R, Tripathy I, Hedgecock S, Fowler-Taylor A, Shen H and Fox H: Omalizumab, an anti-IgE antibody, in the treatment of adults and adolescents with perennial allergic rhinitis. Ann Allergy Asthma Immunol 91: 160-167, 2003.

4. Potter PC, van Niekerk CH and Schoeman HS: Effects of triamcinolone on quality of life in patients with persistent allergic rhinitis. Ann Allergy Asthma Immunol 91: 368-374, 2003.

5. Ciprandi G, Tosca MA, Cirillo I and Vizzaccaro A: The effect of budesonide on the cytokine pattern in patients with perennial allergic rhinitis. Ann Allergy Asthma Immunol 91: 467-471, 2003.

6. Pearlman DS, Lumry WR, Winder JA and Noonan MJ: Oncedaily cetirizine effective in the treatment of seasonal allergic rhinitis in children aged 6 to 11 years: a randomized, doubleblind, placebo-controlled study. Clin Pediatr 36: 209-215, 1997.

7. Hordijk GJ, Antvelink JB and Luwema RA: Sublingual immunotherapy with a standardized grass pollen extract; a double-blind placebo-controlled study. Allergol Immunopathol 26: 234-240, 1998.

8. Bernstein DI, Bernstein CK, Deng C, Murphy KJ, Bernstein IL, Bernstein JA and Shukla R: Evaluation of the clinical efficacy and safety of grapeseed extract in the treatment of fall seasonal allergic rhinitis: a pilot study. Ann Allergy Asthma Immunol 88: 272-278, 2002.

9. Takano H, Osakabe N, Sanbongi C, Yanagisawa R, Inoue K, Yasuda A, Natsume M, Baba S, Ichiishi E and Yoshikawa T: Extract of Perilla frutescens enriched for rosmarinic acid, a polyphenolic phytochemical, inhibits seasonal allergic rhinoconjunctivitis in humans. Exp Biol Med 229: 247-254, 2004.

10. Kobayashi M, Matsushita H, Tsukiyama R, Saito M and Sugita T: Shoyu polysaccharides from soy sauce improve quality of life for patients with seasonal allergic rhinitis: a double-blind placebocontrolled clinical study. Int J Mol Med 15: 463-467, 2005.
11. Murakami A, Nakamura Y, Torikai K, Tanaka T, Koshiba T, Koshimizu K, Kuwahara S, Takahashi Y, Ogawa K, Yano M, Tokuda H, Nishino H, Mimaki Y, Sashida Y, Kitanaka S and Ohigashi $\mathrm{H}$ : Inhibitory effect of citrus nobiletin on phorbol ester-induced skin inflammation, oxidative stress, and tumor promotion in mice. Cancer Res 60: 5059-5066, 2000.

12. Sato T, Koike L, Miyata Y, Hirata M, Mimaki Y, Sashida Y, Yano $M$ and Ito $A$ : Inhibition of activated protein-1 binding activity and phosphatidylinositol 3-kinase pathway by nobiletin, a polymethoxy flavonoid, results in augmentation of tissue inhibitor of metalloproteinase-1 production and suppression of production of matrix metalloproteinases 1 and 9 in human fibrosarcoma HT-1080 cells. Cancer Res 62: 1025-1029, 2002.

13. Miyata Y, Sato T, Yano M and Ito A: Activation of protein kinase $\mathrm{C} B \mathrm{II} / \varepsilon$-c-Jun $\mathrm{NH}_{2}$-terminal kinase pathway and inhibition of mitogen-activated protein/extracellular signal-regulated kinase $1 / 2$ phosphorylation in antitumor invasive activity induced by the polymethoxy flavonoid, nobiletin. Mol Cancer Ther 3: 839-847, 2004.

14. Park S-H, Park E-K and Kim D-H: Passive cutaneous anaphylaxisinhibitory activity of flavanones from Citrus unshiu and Poncirus trifoliate. Planta Med 71: 24-27, 2005.

15. Middleton R and Drzewiecki G: Flavonoid inhibition of human basophil histamine release stimulated by various agents. Biochem Pharmacol 33: 3333-3338, 1984.

16. Higashi-Okai K, Kamimoto K, Yoshioka A and Okai Y: Potent suppressive activity of fresh and dried peels from Satsuma mandarin (Citrus unshiu Marcorv.) on hydroperoxide generation from oxidized linoleic acid. Phytother Res 16: 781-784, 2002.

17. Kita M, Komatsu A, Omura M, Yano M, Ikoma Y and Moriguchi T: Cloning and expression of CitPDS1, a gene encoding phytoene desaturase in citrus. Biosci Biotechnol Biochem 51: 7346-7351, 2003.

18. Barsumian EL, Isersky MG, Petrino MG and Siraganian RP: IgE-induced histamine release from rat basophilic leukemia cell lines: isolation of releasing and non-releasing clones. Eur J Immunol 11: 317-322, 1981.

19. Mita H, Yui Y, Yasueda H, Kajita T, Saito H and Shida T: Allergen induced histamine release and immunoreactive-leukotriene $\mathrm{C}_{4}$ generation from leukocytes in mite sensitive asthmatic patients. Prostaglandins 31: 869-886, 1986.

20. Tanabe S, Arai S, Yanagihara Y, Mita H, Takahashi K and Watanabe M: A major wheat allergen has a Gln-Gln-Gln-ProPro motif identified as an IgE-binding epitope. Biochem Biophys Res Commun 219: 290-293, 1996.

21. Saito K, Horie M, Nose N, Nakagomi K and Nakazawa H: High-performance liquid chromatography of histamine and 1methylhistamine with on-column fluorescence derivatization. J Chromatogr 595: 163-168, 1992.

22. Watanabe J, Shinmoto H and Tsushida T: Coumarin and flavone derivatives from estragon and thyme as inhibitors of chemical mediator release from RBL-2H3 cells. Biosci Biotechnol Biochem 69: 1-6, 2005.

23. Demo SD, Masuda E, Rossi AB, Throndset BT, Gerard AL, Chan EH, Armstrong RJ, Fox BP, Lorens JB, Payan DG, Scheller RH and Fisher JM: Quantitative measurement of mast cell degranulation using a novel flow cytometric annexin-V binding assay. Cytometry 36: 340-348, 1999.

24. Choi OH, Kim JH and Kinet JP: Calcium mobilization via sphingosine kinase in signaling by the FceRI antigen receptor. Nature 380: 634-636, 1996.

25. Tedeschi A, Lorini M, Galbiati S, Gibelli S and Miadonna A: Inhibition of basophil histamine release by tyrosine kinase and phosphatidylinositol 3-kinase inhibitors. Int J Immunopharmacol 22: 797-808, 2000.

26. Franke TF, Kaplan DR, Cantley LC and Toker A: Direct regulation of the Akt proto-oncogene product by phosphatidylinositol-3,4-bisphosphate. Science 275: 665-668, 1997.

27. Lee N-K, Choi S-H, Park S-H, Park E-K and Kim D-H: Antiallergic activity of hesperidin is activated by intestinal microflora. Pharmacology 71: 174-180, 2004.

28. Ameer B, Weintraub RA, Johnson JV, Yost RA and Rouseff RL: Flavone absorption after naringin, hesperidin and Citrus administration. Clin Pharmacol Ther 60: 34-40, 1996.

29. Spencer JPE, Chowrimootoo G, Choudhury R, Edward S, Debnam ES, Srai SK and Rice-Evans C: The small intestine can both absorb and glucuronidate luminal flavonoids. FEBS Lett 458: 224-230, 1999. 